\title{
Erratum to: Professional Vision of Classroom Management and Learning Support in Science Classrooms-Does Professional Vision Differ Across General and Content-Specific Classroom Interactions?
}

\author{
Mirjam Steffensky • Bernadette Gold • \\ Manfred Holdynski • Kornelia Möller
}

Received: 20 January 2015 / Accepted: 20 January 2015 /Published online: 5 February 2015

(C) Ministry of Science and Technology, Taiwan 2015

\section{Erratum to: Int $\mathbf{J}$ of Sci and Math Educ DOI 10.1007/s10763-014-9607-0}

The Affiliation for Kornelia Möller should read: Seminar for Elementary Science Education, University of Muenster, Münster, Germany

Steffensky, M., Gold, B., Holodynski, M., \& Möller, K. (2015). Professional Vision of Classroom Management and Learning Support in Science Classrooms-Does Professional Vision Differ Across General and Content-Specific Classroom Interactions? Int J of Sci and Math Educ, (Doi: 10.1007/s10763-014-9607-0)

In the article by Steffensky, Gold, Holodynski, \& Möller (2015) several corrections in Table 1 need to be made. Please replace published version with corrected version below. The editors apologize for this error.

The online version of the original article can be found at http://dx.doi.org/10.1007/s10763-014-9607-0.

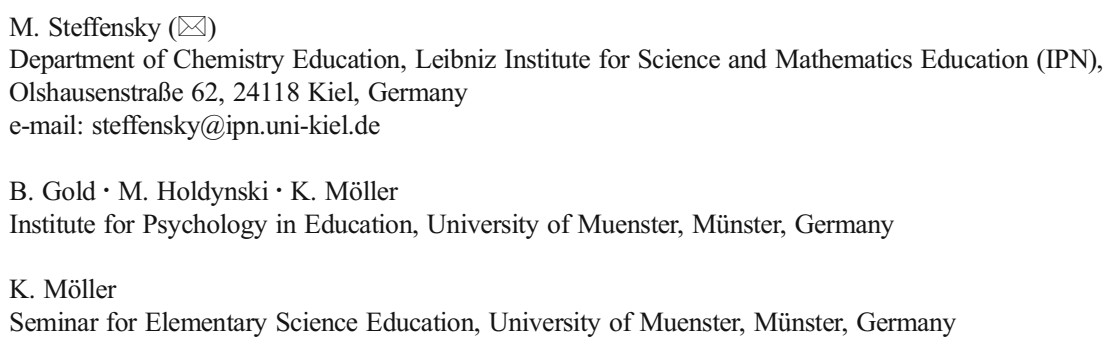


Table 1 Parcel loadings on factors in explorative and confirmatory nested-factor models

\begin{tabular}{|c|c|c|c|c|c|c|c|c|c|}
\hline \multirow{2}{*}{$\begin{array}{l}\text { Parcel } \\
\text { V1 CA }\end{array}$} & \multirow{2}{*}{$\begin{array}{l}\begin{array}{l}\text { Dimen- } \\
\text { sion of } \\
\text { PV }\end{array} \\
\text { PVLS }\end{array}$} & \multirow{2}{*}{$\begin{array}{l}\text { Facet } \\
\mathrm{CA}\end{array}$} & \multirow{2}{*}{$\begin{array}{l}\text { Video } \\
1\end{array}$} & \multicolumn{2}{|c|}{$\begin{array}{l}\text { Exploratory nested- } \\
\text { factor model }\end{array}$} & \multicolumn{4}{|c|}{ Confirmatory nested-factor models } \\
\hline & & & & .412 & .175 & .513 & & .505 & .604 \\
\hline V1 S & PVLS & $\mathrm{S}$ & 1 & .537 & .077 & .572 & & .566 & .677 \\
\hline V2 S1 & PVLS & $\mathrm{S}$ & 2 & .140 & .659 & .536 & & .500 & .445 \\
\hline V2 S2 & PVLS & $\mathrm{S}$ & 2 & .352 & .359 & .564 & & .548 & .472 \\
\hline V3 CA & PVLS & $\mathrm{CA}$ & 3 & .398 & .394 & .631 & & .621 & .550 \\
\hline V3 S & PVLS & $\mathrm{S}$ & 3 & .389 & .333 & .582 & & .577 & .440 \\
\hline V4 CA1 & PVLS & $\mathrm{CA}$ & 4 & .721 & -.029 & .666 & & .694 & .397 \\
\hline V4 CA2 & PVLS & CA & 4 & .600 & -.030 & .546 & & .573 & .400 \\
\hline V5 CA & PVLS & $\mathrm{S}$ & 5 & .702 & .025 & .677 & & .694 & .453 \\
\hline V5 S & PVLS & $\mathrm{S}$ & 5 & .746 & -.145 & .602 & & .625 & .434 \\
\hline V6 CA & PVLS & $\mathrm{CA}$ & 6 & .744 & -.006 & .699 & & .721 & .516 \\
\hline V6 S & PVLS & $\mathrm{S}$ & 6 & .686 & .042 & .672 & & .689 & .452 \\
\hline V7 MO & PVCM & MO & 7 & .298 & .152 & .395 & .476 & & .665 \\
\hline V7 MM & PVCM & MM & 7 & .188 & .207 & .318 & .382 & & .560 \\
\hline V7 RR & PVCM & $\mathrm{RR}$ & 7 & .253 & .282 & .435 & .560 & & .612 \\
\hline V8 MO & PVCM & MO & 8 & .030 & .488 & .332 & .435 & & .773 \\
\hline V8 MM & PVCM & MM & 8 & -.004 & .567 & .250 & .299 & & .507 \\
\hline V8 RR & PVCM & $\mathrm{RR}$ & 8 & -.031 & .453 & .349 & .450 & & .657 \\
\hline V9 MO & PVCM & MO & 9 & .170 & .301 & .364 & .612 & & .445 \\
\hline V9 RR & PVCM & $\mathrm{RR}$ & 9 & .283 & .105 & .347 & .459 & & .432 \\
\hline V10 MO & PVCM & MO & 10 & -.044 & .508 & .276 & .422 & & .731 \\
\hline V10 MM & PVCM & MM & 10 & -.001 & .528 & .334 & .532 & & .671 \\
\hline V10 RR & PVCM & $\mathrm{RR}$ & 10 & -.063 & .523 & .270 & .421 & & .573 \\
\hline
\end{tabular}

In the exploratory nested-factor model highest or similiar loadings are highlighted

$P V$ Professional vision, $P V L S$ Professional vision learning support, $P V C M$ Professional vision of classroom management, $C A$ Cognitive activation, $S$ Structuring, $M O$ Monitoring, $M M$ Managing Momentum, $R R$ Rules and Routines 